\title{
Preclinical evaluation of a urokinase plasminogen activator receptor-targeted nanoprobe in rhesus monkeys
}

This article was published in the following Dove Press journal:

International Journal of Nanomedicine

28 October 2015

Number of times this article has been viewed

\author{
Yushu Chen' \\ Li Gong ${ }^{2}$ \\ Ning $\mathrm{Gao}^{3}$ \\ Jichun Liao' \\ Jiayu Sun' \\ Yuqing Wang' \\ Lei Wang' \\ Pengjin Zhu' \\ Qing Fan' \\ Yongqiang Andrew Wang ${ }^{4}$ \\ Wen Zeng ${ }^{2}$ \\ Hui $\mathrm{MaO}^{3}$ \\ Lily Yang ${ }^{5}$ \\ Fabao Gao' \\ 'Molecular Imaging Center, \\ Department of Radiology, West China \\ Hospital, Sichuan University, Chengdu, \\ ${ }^{2}$ Sichuan Primed Bio-Tech Group Co, \\ Ltd, Chengdu, People's Republic of \\ China; ${ }^{3}$ Department of Radiology and \\ Imaging Sciences, Emory University \\ School of Medicine, Atlanta, GA, \\ ${ }^{4}$ Ocean NanoTech, LLC, San Diego, \\ CA, ${ }^{5}$ Department of Surgery, Emory \\ University School of Medicine, Atlanta, \\ GA, USA
}

Correspondence: Fabao Gao Department of Radiology, West China Hospital, Sichuan University, 37 Guoxuexiang, Chengdu, Sichuan 61004I, People's Republic of China Tel +8628 85I 6408 I

Fax +86 28 8542 2I35

Email gaofabao@yahoo.com
Purpose: To translate a recombinant peptide containing the amino-terminal fragment (ATF) of urokinase plasminogen activator receptor-targeted magnetic iron oxide (IO) nanoparticles (uPAR-targeted human ATF-IONPs) into clinical applications, we conducted a pilot study to evaluate the toxicity and pharmacokinetics of this nanoparticle in normal rhesus monkeys.

Methods: We assessed the changes in the following: magnetic resonance imaging (MRI) signals from pretreatment stage to 14 days posttreatment, serum iron concentrations from 5 minutes posttreatment to 12 weeks posttreatment, routine blood examination and serum chemistry analysis results from pretreatment stage to 12 weeks after administration, and results of staining of the liver with Perls' Prussian Blue and hematoxylin-eosin at 24 hours and 3 months posttreatment in two rhesus monkeys following an intravenous administration of the targeted nanoparticles either with a polyethylene glycol (ATF-PEG-IONP) or without a PEG (ATF-IONP) coating.

Results: The levels of alkaline phosphatase, alanine transaminase, and direct bilirubin in the two monkeys increased immediately after the administration of the IONPs but returned to normal within 20 days and stayed within the normal reference range 3 months after the injection. The creatinine levels of the two monkeys stayed within the normal range during the study. In addition, red blood cells, white blood cells, hemoglobin level, and platelets remained normal during the 3 months of the study.

Conclusion: All of the results suggest that a transient injury in terms of normal organ functions, but no microscopic necrotic lesions, was observed at a systemic delivery dose of $5 \mathrm{mg} / \mathrm{kg}$ of iron equivalent concentration in the acute phase, and that no chronic toxicity was found 3 months after the injection. Therefore, we conclude that uPAR-targeted IONPs have the potential to be used as receptor-targeted MRI contrasts as well as theranostic agents for the detection and treatment of human cancers in future studies.

Keywords: uPAR-IONP, nonhuman primates, transient harm, self-healing

\section{Introduction}

With oncology stepping into the molecular era, there has been an urgent demand for oncologists to understand the key molecular events and their associated biomarkers in both normal and abnormal tissues at the molecular and cellular levels. ${ }^{1-3}$ Molecular imaging, by using noninvasive imaging approaches, has the tenability of the surface of the nanoparticles, which can potentially prolong the circulation of the agent in the blood or accurately target a specific location within the body, ${ }^{4}$ helping to detect tumors and identify biomarker expression in tumors. The past decade has witnessed an unprecedented expansion of the engineering of theranostic nanoparticles for cancer imaging and therapy. ${ }^{5}$ However, the side/toxicological effects of the nanoparticles on health have aroused considerable and increasing concerns worldwide. ${ }^{6}$ An increasing number 
of toxicological investigations of nanoparticles have revealed that nanoparticles tend to exhibit toxicological effects in vivo, ${ }^{6,7}$ fairly different from the larger particles of the same chemical composition (on an identical mass basis). In addition, most toxicity studies of nanoparticles have been focused on the acute or subacute phase in small animal models, ${ }^{6,8,9}$ whereas only a few studies have investigated the toxicity of nanoparticles to humans ex vivo. ${ }^{10}$ For example, the United States Food and Drug Administration approved only the use of liposome-encapsulated doxorubicin and paclitaxel attached to nanoparticles in cancer therapy. ${ }^{11,12}$ Thus, obviously, it is imperative that a toxicity investigation of superb nanoparticles from basic research to clinical application be conducted in the shortest possible time.

An elevated level of urokinase plasminogen activator receptor (UPAR) has been found in cancers of many types, and its expression correlates with a poorer prognosis, thus providing a useful predictor of high-risk patients. ${ }^{13-18}$ Blocked uPAR pathway can reduce angiogenesis and inhibit primary tumor growth or metastasis in various models in vivo. ${ }^{13,19-21}$ Increasing evidence has proved the excellent performance of a novel cell surface receptor-targeted magnetic resonance imaging (MRI) nanoprobe in targeted cancer imaging and therapy; this nanoprobe is produced by the conjugation of a recombinant amino-terminal fragment (ATF) peptide of the urokinase plasminogen activator (uPA) to magnetic iron oxide nanoparticles (IONPs). IONPs can surmount the tumor's stromal barrier and increase the intracellular drug concentration in pancreatic or breast cancer cells and tumor environment while producing MRI contrast to monitor drug delivery and therapeutic response. ${ }^{22}$ Moreover, previous studies by Yang et $\mathrm{al}^{18}$, Liu et $\mathrm{al}^{19}$, and Lee et $\mathrm{al}^{22}$ have shown the ideal diagnostic and therapeutic capabilities of the uPARIONP and there were no apparent damages detected to other organs and tissues following systemic delivery. Given that animals such as rodents differ significantly from humans in size, genetic relationship, functions, and structures, ${ }^{23,24}$ preclinical studies in large animal models is of great necessity to determine systemic toxicity, biodistributions, dose, imaging capability, and pharmacokinetics (PK), to translate this receptor-targeted MRI probe into clinical applications for cancer detection and image-guided cancer therapy. Compared with other experimental animals, rhesus monkeys are generally considered a preclinical model closest to humans ${ }^{25,26}$ and the PK studies conducted in the primate are highly applicable to predict the PK in humans. ${ }^{27}$

In this study, we examined the toxicity and PK of uPARtargeted human ATF-conjugated IONPs in normal rhesus monkeys. We further evaluated the feasibility of noninvasive MRI monitoring of IONP accumulation in the major organs following systemic delivery. To our knowledge, the current study is the first preclinical toxicity evaluation of a targeting ligand-conjugated magnetic IONP in nonhuman primary animals, and there have been no reports on the PK of liver, kidney, and cardiac functions for the 3 months after intravenous administration of the IONPs.

\section{Materials and methods Preparation of uPAR-targeted ATF-IONPs} Magnetic IONPs of $10 \mathrm{~nm}$ core size were obtained from Ocean Nanotech, LLC, San Diego, CA, USA. The IONPs coated with amphiphilic polymers, with or without an additional layer of polyethylene glycol (PEG), were conjugated with human ATF peptide produced by a bacterial expression system via a covalent bond between carboxyl groups of the amphiphilic polymers and amino side groups of the peptides at a ratio of 1 IONP:15 ATF peptides. The final ATF-IONP or ATF-PEG-IONP conjugates were purified using Nanosep $100 \mathrm{~K}$ column. The testing of the targeted-ATF-IONPs or ATF-PEG-IONPs showed that they were free of endotoxin contamination.

\section{Preparation of experimental animals}

Two healthy young rhesus monkeys ( $4 \pm 1$ years old; $3.8 \pm 0.1 \mathrm{~kg}$; both males) were obtained from Sichuan Primed Bio-Tech Group Co, Ltd, Chengdu, People's Republic of China. They were housed individually in the West China Experimental Animal Center at Sichuan University, Chengdu, People's Republic of China, and kept under a 12-hour light/ dark cycle. After intravenous anesthetic administration using $3-5 \mathrm{~mL}$ of $3 \%$ pentobarbital sodium at $2 \mathrm{~mL} / \mathrm{min}$, the animals received intravenous administration of the uPAR-targeted human ATF-IONPs. Monkey 1 received intravenous administration of the $5 \mathrm{mg} / \mathrm{kg}$ of Fe equivalent dose of human ATFIONP and Monkey 2 received $5 \mathrm{mg} / \mathrm{kg}$ of Fe equivalent dose of human ATF-PEG-IONP. The monkeys were maintained in conformity with the requirements of the National Institutes of Health's Guide for the Care and Use of Laboratory Animal of the USA, and all experimental protocols were reviewed and approved by the Institutional Animal Care and Use Committee of Sichuan PriMed Bio-Tech Group Co, Ltd.

\section{MRI parameters}

MRI scan was performed on a clinically available 3.0 T Siemens Trio system (Siemens, Erlangen, Germany) with contiguous electrocardiography and breath-hold monitoring 
of the rhesus monkeys, under anesthetic administration using $15 \mathrm{mg} / \mathrm{kg}$ intravenous pentobarbital sodium at $2 \mathrm{~mL} / \mathrm{min}$. MRI scan using a surface coil was performed on the monkeys before and at 10 minutes, 30 minutes, 60 minutes, 1 day, 7 days, and 14 days after intravenous IONP administration. T2-weighted imaging (T2WI) (repetition time $=3,200 \mathrm{~ms} / \mathrm{echo}$ time $=65 \mathrm{~ms}$; slices $=15$, slice thickness $=1 \mathrm{~mm}$, averages $=3$ ) and T1-weighted imaging (T1WI) (repetition time $=500 \mathrm{~ms} /$ echo time $=12.0 \mathrm{~ms}$; slices $=25$, slice thickness $=1 \mathrm{~mm}$, averages $=6$ ) were conducted. The region of interest method was used to assess the IONP-induced changes in MRI signal level, contrast, and T2 values in the liver and the spleen. Signals of the back muscles, showing little change before and after the injection of ATF-IONPs, were used as a baseline to compare the signals in the regions of interest.

\section{Serum iron concentrations and systemic toxicity}

To quantify IONPs in the blood following systemic delivery, blood samples were collected at 5 minutes; 1 hour, 3 hours, 6 hours, 12 hours, 24 hours, and 48 hours; 4 days and 10 days; and 2 weeks, 4 weeks, and 12 weeks after the injections with the monkeys under anesthesia. Serum plasma samples were digested in acid. The resulting solutions were analyzed by inductively coupled plasma mass spectrometry (ICP-MS) to determine the amount of $\mathrm{Fe}$ in the blood, which is regarded to be representative of the amount of IONPs. To evaluate the toxicity of the IONPs to the liver and the other normal organs, as well as the hematology, we collected blood samples at different intervals following IONP administration and then determined serum levels of alanine transaminase (ALT), alkaline phosphatase (ALP), aspartate transaminase (AST), and direct bilirubin (BIL-D) for the liver function, creatinine (CREA) for the kidney function, and creatine kinase (CK) for the cardiac function. Routine blood examinations - mostly estimation of levels of red blood cells (RBCs), white blood cells (WBCs), hemoglobin (HGB), and platelets (PLT) were conducted 7 days before and 2 days, 2 weeks, 4 weeks, 8 weeks, and 12 weeks posttreatment. The blood samples were collected under sterile conditions in heparinized tubes for hematology and nonheparinized tubes for biochemistry. Vital signs, body weight, appetite, and movements of the monkeys were monitored daily.

\section{Perls' Prussian Blue and hematoxylin- eosin staining}

Biopsy samples of the liver were obtained from the rhesus monkeys at 24 hours and 3 months after the IONP injection using core needle biopsy after MRI scanning and blood collected with the monkeys under anesthesia. The samples were placed in $10 \%$ formalin, then fixed with $4 \%$ formalin solution, and were stained with hematoxylin-eosin (HE) solution using the standard protocol. HE staining of the normal liver was included in this study as a negative control, and Prussian Blue staining was used to confirm the presence of IONPs in the liver tissue sections using our established method. ${ }^{17}$ Microscopy was used to determine acute inflammatory changes or necrotic areas associated with the IONP administration and for highlighting the iron deposits in Kupffer cells of the sinusoids of the liver tissue sections.

\section{Results}

\section{Noninvasive MRI monitoring of the delivery of the IONPs}

It was found that magnetic nanoparticles typically lead to reduced MRI signals during T2WI in the area where nanoparticles accumulate (Figure 1A and B). The signal intensity of T2WI signal was found to have decreased by $27 \%$ and $29 \%$ in the liver of monkeys 1 and 2, respectively, following an intravenous delivery of human ATF-IONPs and human ATFPEG-IONPs for 10 minutes. The T2 signal then remained relatively low until 14 days after the administration, with gradually increasing signal intensity thereafter (Figure 1C). The liver of Monkey 2 that received human ATF-PEG-IONPs appeared to show $\mathrm{T} 2$ signal intensity higher than that receiving ATF-IONPs without PEG coating in the liver, thus indicative of a possible effectively reduced nonspecific uptake of the ATF-PEG-IONPs in the liver or higher bioavailability of ATF-PEG-IONP in the plasma (Figure 1C). Similarly, the T2 signal intensity in the spleen decreased in both monkeys after the administration and remained relatively low until 14 days posttreatment (Figure 1D). In addition, the T1 signal intensity in the liver and the spleen also diminished immediately after the IONP administration and remained at a low level 14 days later (Figure 1E and F).

\section{Toxicity and PK of ATF-IONPs and ATF- PEG-IONPs following systemic delivery}

Following the systemic administration of $5 \mathrm{mg} / \mathrm{kg}$ of iron equivalent concentrations of ATF-IONPs or ATF-PEGIONPs in the monkeys, no changes were found in the vital signs, body weight, appetite, and movements of the monkeys.

The levels of ALP, ALT, AST, and BIL-D values, which represent the liver function, immediately increased 

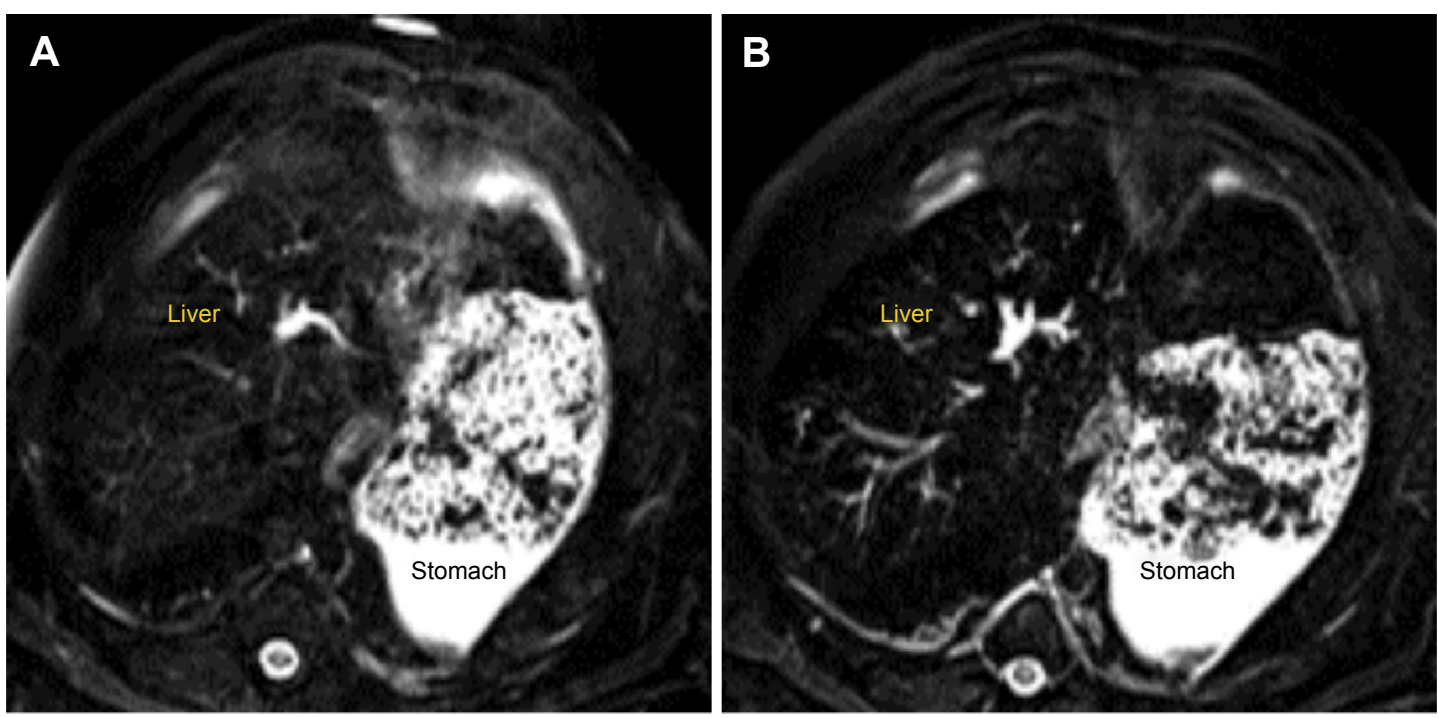

C

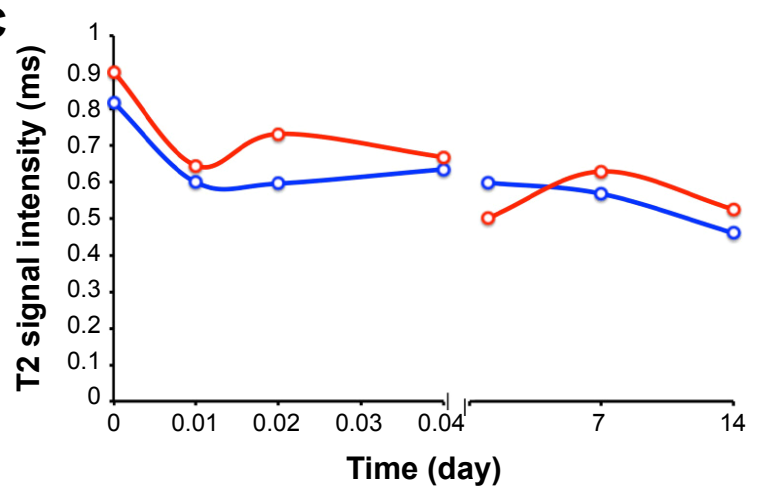

E

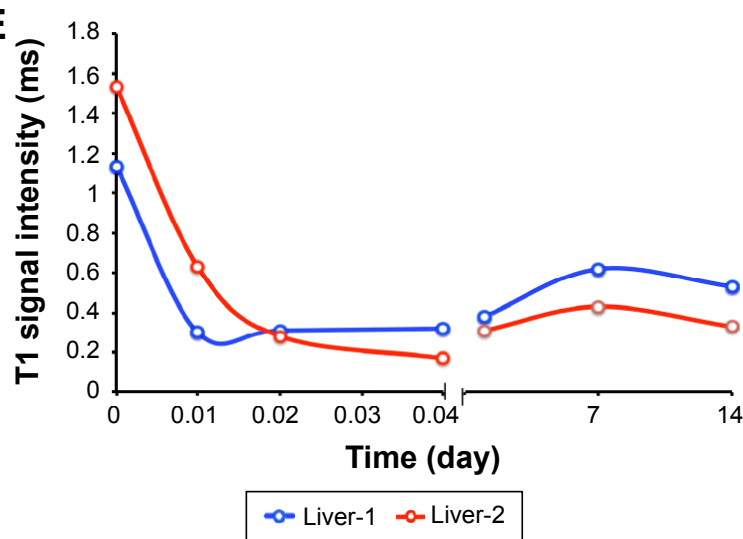

D

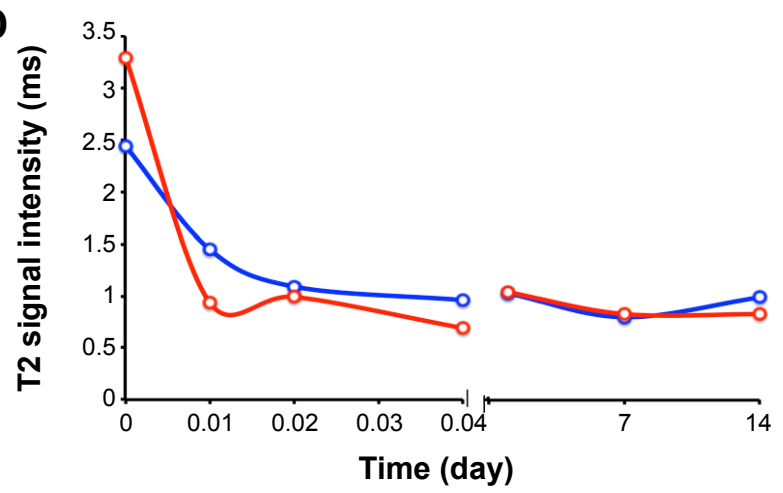

$\mathbf{F}$

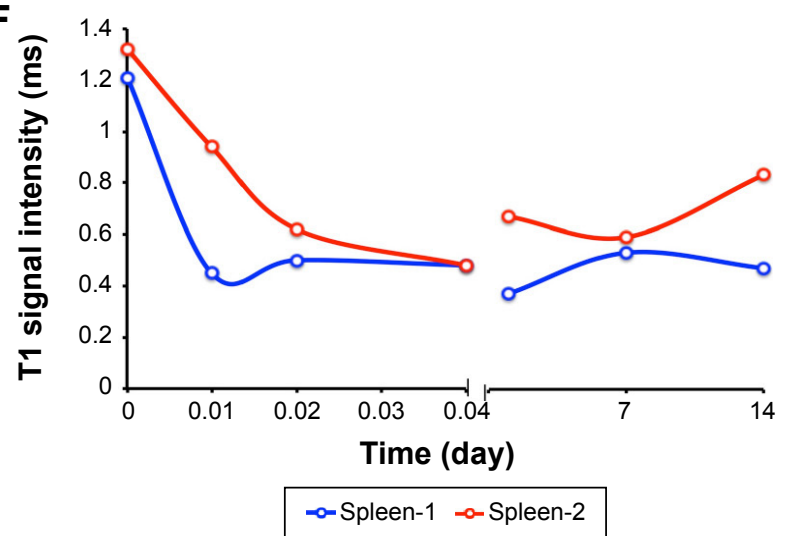

Figure I The MRI signal alterations in liver and spleen.

Notes: (A and B) are the MRI images before and after ATF-IONP injection, respectively, without and with PEG coating, both of them are phased from up to down. (C and D) are the variations of T2 signal intensity; (E and F) are the variations of TI signal intensity. Monkey I received ATF-IONP, and Monkey 2 received ATF-PEG-IONP. Abbreviations: ATF, amino-terminal fragment; IONP, magnetic iron oxide nanoparticle; MRI, magnetic resonance imaging; PEG, polyethylene glycol.

after administration of ATF-PEG-IONPs in Monkey 2. A noticeable increase in the level of ALT value was seen in Monkey 1, which received ATF-IONP. However, the levels of these enzymes returned to normal within 20 days (Figure $2 \mathrm{D}-\mathrm{G})$. Both monkeys had normal renal function because CREA levels were in the normal range during the study (Figure 2I). Monkey 1 injected with ATF-IONP had a normal
CK value, which is regarded as representative of a normal cardiac function. However, Monkey 2 had an abnormal CK value before the nanoparticle injection and $\mathrm{CK}$ value further increased slightly after the ATF-PEG-IONP injection but returned to normal within 20 days (Figure $2 \mathrm{H}$ ). Therefore, there is no knowing whether the increased CK level was caused by the nanoparticle injection or some other factors. 


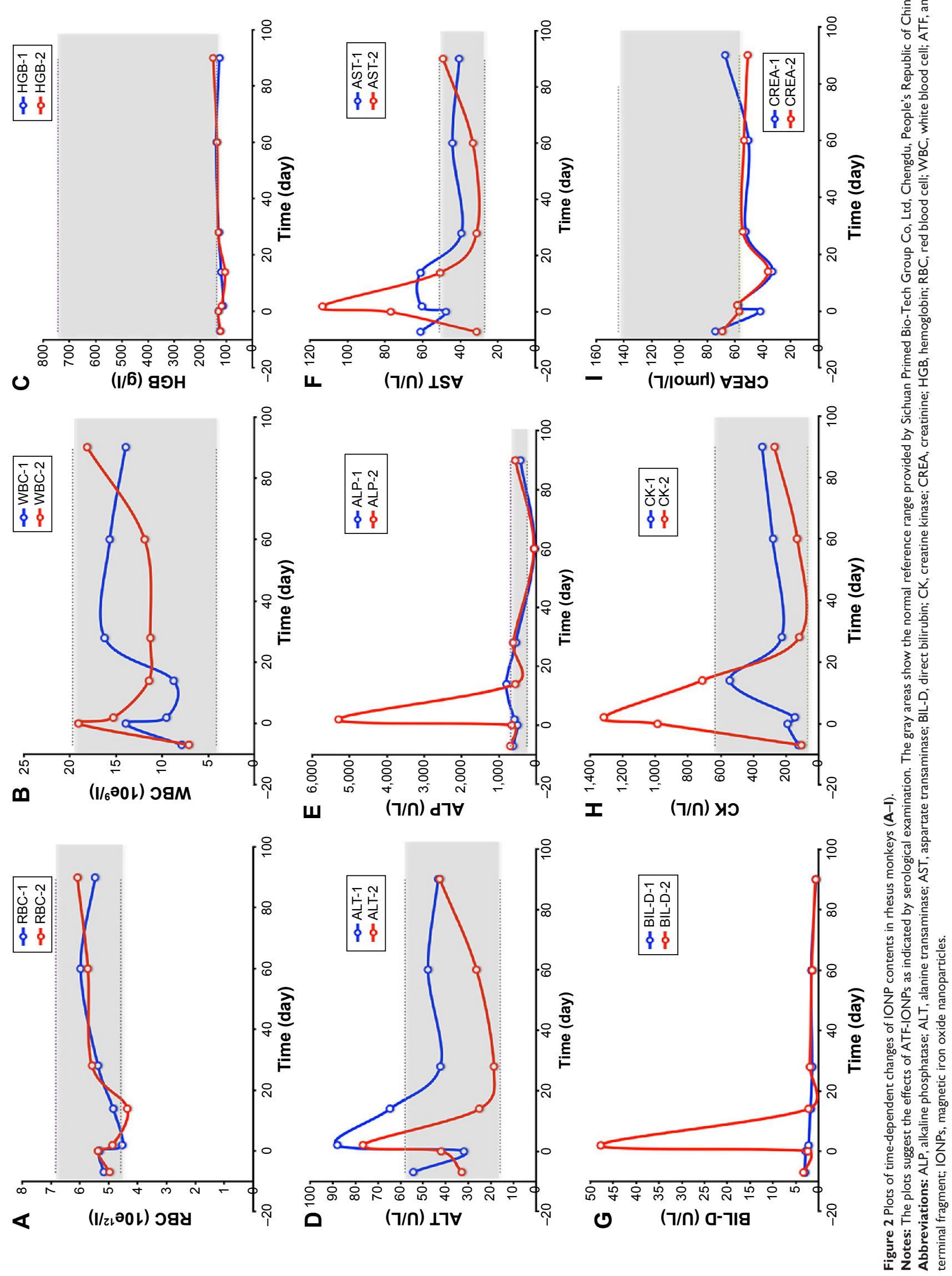


Fortunately, 3 months after the probe injection, we found that most of these figures had recovered to within the normal range, compared with the normal reference range provided from testing $>100$ normal rhesus monkeys by the Sichuan Primed Bio-Tech Group Co, Ltd. Levels of RBCs, WBCs, HGB, and PLT were normal during the 3 months of the study (Figure 2A-C). Therefore, we suggest that uPARtargeted IONPs have been proven to be harmless to rhesus monkeys.

Moreover, it is necessary to determine PK and biodistribution in normal animals for clinical translation of nanoparticle imaging and therapeutic agents. Serum plasma iron level detected by ICP-MS is regarded to be representative of IONP level in the blood. After iron concentrations of the plasma samples were determined by ICP-MS, the values were used to calculate PK. Although a similar half-life $T_{1 / 2}$ was found (237-278 hours) in the monkeys that received two different IONPs, it seems that, in the ATF-PEG-IONPinjected monkey, the area under the curve, an indicator of IONP bioavailability in plasma, was much larger than that in the ATF-IONP infected-monkey (Figure 3). Furthermore, the maximum serum concentration $C_{\text {max }}(821 \mathrm{mg} / \mathrm{L})$ was higher in the ATF-PEG-IONP-injected monkey (Monkey 2) than in the ATF-IONP-injected monkey $(311 \mathrm{mg} / \mathrm{L})$.
Nanoparticle

Animal

Dose

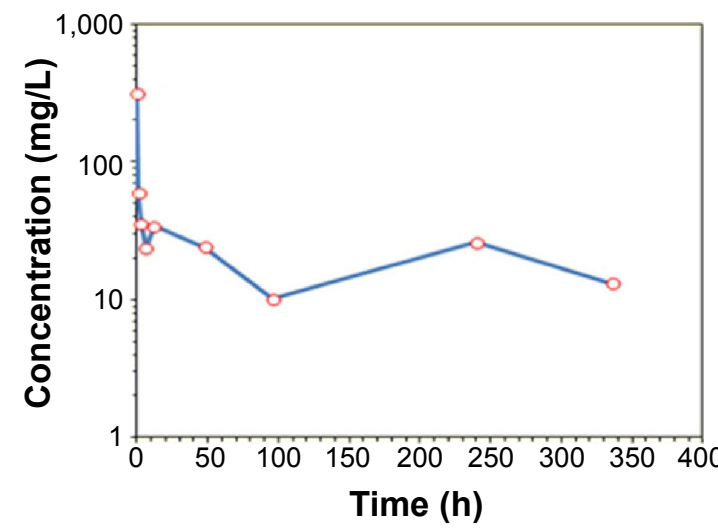

Nanoparticle

Animal

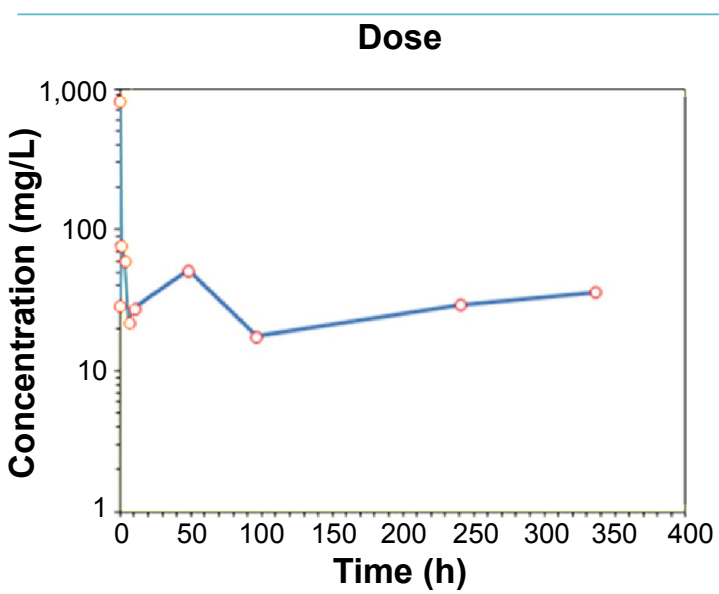

Human ATF-IONP

Rhesus monkey

$5 \mathrm{mg} / \mathrm{kg}$

\begin{tabular}{lll} 
Parameter & Unit & Value \\
$T_{1 / 2}$ & hour & 278.2331172 \\
\hline$T_{\max }$ & hour & 0.083 \\
\hline$C_{\max }$ & $\mathrm{mg} / \mathrm{L}$ & 311.6618 \\
\hline$C_{0}$ & $\mathrm{mg} / \mathrm{L}$ & 362.3247247 \\
\hline $\mathrm{AUC}_{0-\mathrm{t}}$ & $\mathrm{mg} / \mathrm{L} \times \mathrm{h}$ & $6,894.590345$ \\
\hline $\mathrm{MRT}_{\text {0-infobs }}$ & $\mathrm{hour}$ & 410.971754 \\
\hline $\mathrm{Cl}_{\text {_obs }}$ & $\mathrm{L} /\left(\mathrm{kg}{ }^{*} \mathrm{~h}\right)$ & 0.000410728 \\
\hline Vss_obs & $\mathrm{L} / \mathrm{kg}$ & 0.168797493 \\
\hline & &
\end{tabular}

Human ATF-PEG-IONP

Rhesus monkey

$5 \mathrm{mg} / \mathrm{kg}$

\begin{tabular}{lll} 
Parameter & Unit & Value \\
$T_{1 / 2}$ & hour & 236.9590973 \\
\hline$T_{\max }$ & hour & 0.083 \\
\hline$C_{\max }$ & $\mathrm{mg} / \mathrm{L}$ & 821.7139 \\
\hline$C_{0}$ & $\mathrm{mg} / \mathrm{L}$ & 1017.196 \\
\hline $\mathrm{AUC}_{0-t}$ & $\mathrm{mg} / \mathrm{L} \times \mathrm{h}$ & $10,580.89715$ \\
\hline $\mathrm{MRT}_{\text {0-infobs }}$ & $\mathrm{hour}$ & 441.2498988 \\
\hline $\mathrm{Cl}_{-}$obs & $\mathrm{L} /\left(\mathrm{kg}{ }^{*} \mathrm{~h}\right)$ & 0.000216979 \\
\hline Vss_obs & $\mathrm{L} / \mathrm{kg}$ & 0.095741978 \\
\hline
\end{tabular}

Figure 3 Determination of PK of uPAR-targeted IONPs in rhesus monkeys.

Notes: After the administration of IONPs, the concentrations of probes change with time elapsed. Upper panel: A monkey received 5 mg/kg of ATF-IONP. Lower panel: A monkey received $5 \mathrm{mg} / \mathrm{kg}$ of ATF-PEG-IONP.

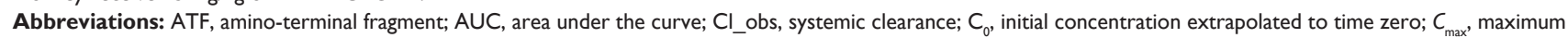
serum concentration; IONPs, magnetic iron oxide nanoparticles; PEG, polyethylene glycol; PK, pharmacokinetics; MRT, mean residence time; $T_{1 / 2}$, half-life; uPAR, urokinase plasminogen activator receptor; Vss_obs, apparent volume of distribution at steady state. 


\section{Histology}

Prussian Blue staining of IONPs in the tissue sections confirmed that ATF-IONPs had accumulated in the liver for $>3$ months after the injection (Figure 4). However, the level of IONPs at 3 months was found to be significantly lower in the liver than that at 1 day after the injection. HE staining was performed to determine the histological changes in the liver. Compared with the normal liver, monkeys 1 and 2 showed swelling of hepatocytes but minimal hepatic necrotic lesions in the tissue sections at 24 hours posttreatment and subsequent recovery to normal histological appearances of hepatocytes and the sinusoidal and bile ductal structures at 3 months posttreatment, except that little swelling of hepatocytes was found in Monkey 1, compared with the negative control, which were verified by tests by West China Frontier Pharma Tech Co, Ltd, Chengdu, Sichuan, People's Republic of China (Figure 5).

\section{Discussion}

Previous studies have shown the feasibility of producing nanoparticle-based imaging probes for in vivo MRI of cancers, ${ }^{14,28}$ and the impacts of nanoparticles on small animals have been investigated as well. ${ }^{6,8,9}$ Nevertheless, scarce data

\section{4 hours}
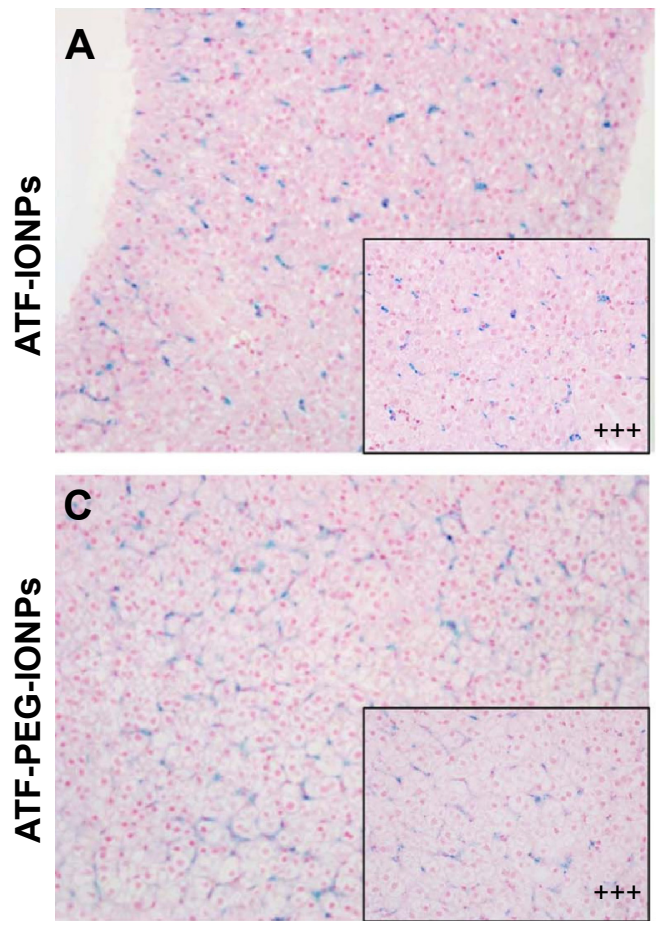

on humans or large animals have been published previously. The current report summarizes extended observations of the PK and safety of the rhesus monkey model. It allows us to refine the diagnosis or cancer therapy strategy, may support long-term treatment of cancer-bearing monkeys, and may aid in predicting probable profiles in humans.

It has been acknowledged that nanoparticles can be taken up by the reticuloendothelial system in the liver and the spleen and are subsequently metabolized or utilized for iron storage.$^{29,30}$ Earlier investigations have shown that MRI contrast enhancement by magnetic nanoparticles is highly related to their composition, size, surface properties, and degree of aggregation in the biological environment. ${ }^{31,32}$ The competence of nanoparticles for MRI signal enhancement correlates directly with the particle size,${ }^{33}$ but it becomes less significant for sizes of single-core IONPs ranging $>50 \mathrm{~nm} .{ }^{34}$ Previous investigations have found that intravenous injection of UPAR-IONP probes resulted in nonspecific uptake by the Kupffer cells in the liver and macrophages in the spleen rather than in other vital organs. Similarly, results of this research have demonstrated that after the intravenous administration of the $10 \mathrm{~nm}$ core size magnetic IONPs, the T1 and T2 signal intensities for the liver and the spleen decreased, which

\section{3 months}
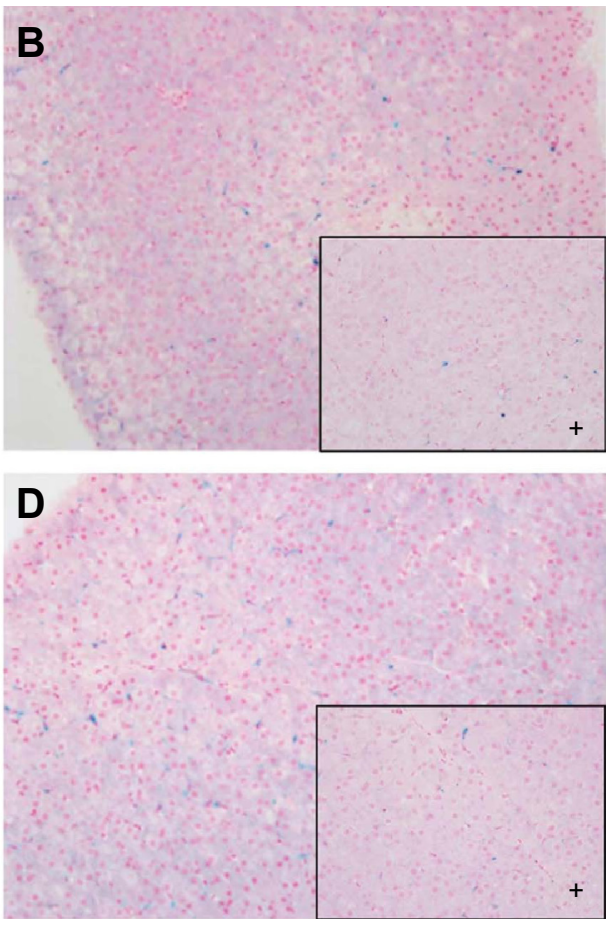

Figure 4 Detection of IONPs in the livers of rhesus monkeys by Prussian Blue staining

Notes: Original magnification: $\times 200$; magnified to $\times 400$ in black box. (A and C) are the biopsy samples of the liver obtained at 24 hours and massive 1 ONPS seen in the kupffer cells $(+++)$, and (B and $\mathbf{D})$ are samples obtained at 3 months after the IONP injection, and sparse IONPs seen in the kupffer cells (+). The liver of Monkey 2 has weaker blue staining compared with that of Monkey I (A and C, respectively). And the levels of IONPs in the liver of monkeys are significantly decreased at 3 months posttreatment. No significant difference between (B and $\mathbf{D})$ was observed.

Abbreviation: IONPs, magnetic iron oxide nanoparticles. 

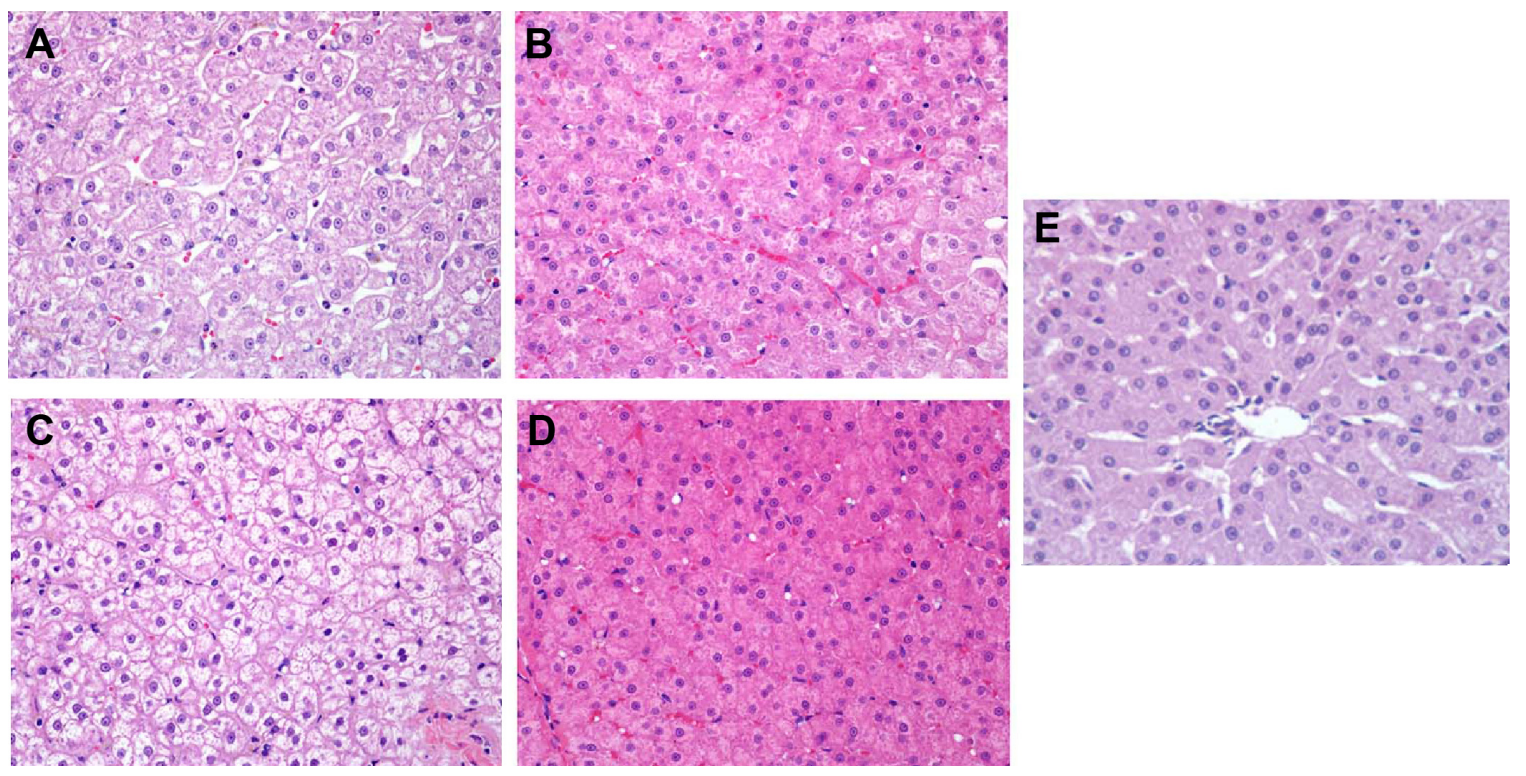

Figure 5 Effects of IONPs in the liver of the rhesus monkeys by H\&E staining.

Notes: Original magnification: $\times 400$. (A and B) are the liver samples of Monkey I at 24 hours and 3 months posttreatment, respectively. (C and $\mathbf{D})$ are the liver samples of Monkey 2 at 24 hours and 3 months posttreatment, respectively. Obvious swelling of hepatocytes and minimal necrosis were seen at 24-hour posttreatment stage, as well as recovery to normal with little swelling of hepatocytes in Monkey I at the 3-month posttreatment stage, compared with the negative control. No significant differences between ( $\mathbf{A}$ and $\mathbf{C}$ ) and between (B and $\mathbf{D}$ ) were observed. (E) is the negative control.

Abbreviations: IONPs, magnetic iron oxide nanoparticles; H\&E, hematoxylin-eosin.

was consistent with our preliminary report that the magnetic IO nanocrystal used in this study can shorten $\mathrm{T} 2$ relaxation time and decrease T1 signal intensity. ${ }^{22}$ This nonspecific accumulation of IONPs in the liver and the spleen of normal monkeys provides us with an opportunity to determine the accumulated MRI signal of ATF-IONPs or ATF-PEG-IONPs in the target organs in vivo, also helping us to determine the feasibility of the MRI scan parameters for use in large animals or future MRI in humans using clinically available 3 T MRI scanners.

More importantly, results of our study show that the blood chemistry variables such as ALT, ALP, BIL-D, and CREA, which are very sensitive to any disease process of the liver ${ }^{35}$ or kidney and which can effectively characterize the restored organs' function, were transiently changed after the IONP administration. Extensive studies have shown that deterioration in the liver function occurs with age and hepatic abnormality is associated with the reduced capacity of the system to metabolize drugs, particularly drugs undergoing oxidative biotransformation. ${ }^{36}$ Concepcion Navarro et al ${ }^{37}$ found that after using arsenic, increment in the activities of AST and ALT in the serum may be related to the leakage of these enzymes from the liver cytosol into the blood stream, which might be an inflammatory response as AST and ALT are reliable determinants of liver parenchymal injury. ${ }^{38,39}$ What is more, the accumulation of nanoparticles in organs such as liver, kidneys, spleen, lung, brain, and heart is closely related to the coefficients of the organs and the inflammatory responses of animals. The small size and large surface areas of nanoparticles endow them with an active group or intrinsic toxicity. ${ }^{9}$ Published data show that smaller nanoparticles $(<25 \mathrm{~nm})$ of $\mathrm{TiO}_{2}$ and $\mathrm{ZnO}$ were more toxic than larger nanoparticles $(<100 \mathrm{~nm}) .^{40}$ Moreover, Kumari et al ${ }^{8}$ reported that in a span of 28 days, in the nanoparticle-treated animals, AST, ALT and lactate dehydrogenase levels increased in the serum, with simultaneous decreases in the figures for the kidney. These indicate tissue necrosis and the possible leakage of these target enzymes into the blood stream. The alterations might probably result from an adaptive mechanism due to nanoparticle stress or altered expression of various genes by $\mathrm{Fe}$ nanoparticles, ${ }^{41}$ which is indicative of hepatotoxicity in treated rats. You et $\mathrm{al}^{42}$ investigated the cytotoxicity, complement activation, and platelet aggregation of PEG-coated hollow gold nanospheres (PEG-HAuNS, average diameter $=63 \mathrm{~nm}$ ) in vitro and vivo. They found that the levels of deposited HAuNS in tissues indeed decreased but did not completely vanish at 90 days, and the AST and ALT values increased in both sexes at both Day 14 and Day 90. In our study, the levels of ALP2, ALT, and BIL-D values increased immediately after the IONP administration, which might be a common phenomenon caused by nanoparticles. With self-healing within a short time period, the levels of these enzymes returned to normal within 20 days and remained in the normal reference range 3 months after the 
probe injection. Both monkeys showed normal renal function because the CREA levels were within the normal range during the study. In addition, Sarhan and Hussein ${ }^{43}$ showed that disrupted blood parameters, such as WBC and HGB levels, were observed after silver nanoparticles (AgNPs) were injected intraperitoneally in rats. However, in our work, we found that levels of RBCs, WBCs, HGB, and PLT remained normal during the 3 months of the study. Moreover, swelling of hepatocytes but minimal hepatic necrotic lesions were observed in both the monkeys at 24 hours posttreatment in our work, as well as recovery to normal histological characteristics of hepatocytes and the sinusoidal and bile ductal structure at 3 months posttreatment, except that there was little swelling of hepatocytes in Monkey 1. The swelling of hepatocytes indicated that in the acute phase, the nanoparticles might affect the permeability of the cell membrane in hepatocytes, ${ }^{35}$ which might lead to adaptation of cell transporters. Comparing the pathological alterations in the two monkeys, a superior recovery in Monkey 2 might have resulted from the fact that the PEG was effective in reducing the hepatotoxicity; ${ }^{44}$ however, there might be individual differences as well. Fortunately, in comparison with other studies, we found by histopathology the promising result that the nanoparticles do not promote steatosis and necrosis and only a transient injury to the normal organ functions was indicated by blood biochemistry. ${ }^{35,43}$ These differences in pathological damages and blood parameters might be mainly caused by the different nanoparticles, dosages, and animal species. In other works, the authors chose a larger dose for injection into rodents. ${ }^{35,42,43,45}$ But it should be pointed out that $5 \mathrm{mg} / \mathrm{kg}$ of Fe equivalent dose of human ATF-IONP is enough for theranostic cancer imaging, as shown in our previous studies. ${ }^{17,46}$ Because of the concern for hepatotoxicity, our pilot toxicity evaluation focused on the effect of the IONPs on liver function and histopathology in the rhesus monkeys according to the previous study, whereas renal and cardiac histopathology was not studied. In addition, only two healthy nonhuman primates, non-cancer-bearing monkeys, were included in this study. The short-term injury response of liver or kidney in cancer-bearing monkeys and humans still await detailed exploration. Further studies will have to improve the IONPs to guarantee greater safety and integrate the relationships among particle size, biological effects, and MRI signal before the long-term treatment of cancer-bearing monkeys can be attempted.

\section{Conclusion}

This study indicates that there was a transient injury to the normal organs and functions but no necrosis was observed at a dose of $5 \mathrm{mg} / \mathrm{kg}$ in the acute phase, and the levels of these enzymes returned to normal and remained in the normal reference range 3 months after the probe injection. This has proved the potential of uPAR-targeted IONPs as receptor-targeted MRI contrasts as well as theranostic agents for the detection and treatment of human cancers. On the basis of this pilot study, further studies are warranted for careful assessment to guarantee greater safety in the long-term treatment of cancerbearing monkeys and to predict probable profiles in humans.

\section{Acknowledgments}

The authors are grateful to the National Natural Science Foundation of China (81130027, 81520108014), the National "Twelfth Five-Year" Plan for Science and Technology Support (2012BAI23B08), and the National Basic Research Program of China (973 Program, 2011CB935800).

\section{Author contributions}

All authors contributed toward data analysis, drafting, and revising the paper and agree to be accountable for all aspects of the work.

\section{Disclosure}

The authors report no conflicts of interest in this work.

\section{References}

1. Sullivan DC. Molecular imaging in oncology. Ann Oncol. 2006;17(supp1 10):x287-x292.

2. Xi L, Zhou G, Gao N, et al. Photoacoustic and fluorescence imageguided surgery using a multifunctional targeted nanoprobe. Ann Surg Oncol. 2014;21(5):1602-1609.

3. James ML, Gambhir SS. A molecular imaging primer: modalities, imaging agents, and applications. Physiol Rev. 2012;92(2):897-965.

4. Estelrich J, Sanchez-Martin MJ, Busquets MA. Nanoparticles in magnetic resonance imaging: from simple to dual contrast agents. Int J Nanomedicine. 2015;10:1727-1741.

5. Chen F, Ehlerding EB, Cai W. Theranostic nanoparticles. J Nucl Med. 2014;55(12):1919-1922.

6. Chen Z, Meng H, Xing G, et al. Acute toxicological effects of copper nanoparticles in vivo. Toxicol Lett. 2006;163(2):109-120.

7. Whitehead A, Stallard N. Opportunities for reduction in acute toxicity testing via improved design. Altern Lab Anim. 2004;32(suppl 2): 73-80.

8. Kumari M, Rajak S, Singh SP, et al. Repeated oral dose toxicity of iron oxide nanoparticles: biochemical and histopathological alterations in different tissues of rats. J Nanosci Nanotechnol. 2012;12(3):2149-2159.

9. Liu H, Ma L, Zhao J, et al. Biochemical toxicity of nano-anatase TiO2 particles in mice. Biol Trace Elem Res. 2009;129(1-3):170-180.

10. Sayes CM, Wahi R, Kurian PA, et al. Correlating nanoscale titania structure with toxicity: a cytotoxicity and inflammatory response study with human dermal fibroblasts and human lung epithelial cells. Toxicol Sci. 2006;92(1):174-185.

11. Petrelli F, Borgonovo K, Barni S. Targeted delivery for breast cancer therapy: the history of nanoparticle-albumin-bound paclitaxel. Expert Opin Pharmacother. 2010;11(8):1413-1432.

12. Leonard RC, Williams S, Tulpule A, Levine AM, Oliveros S. Improving the therapeutic index of anthracycline chemotherapy: focus on liposomal doxorubicin (Myocet). Breast. 2009;18(4):218-224. 
13. Ploug M, Østergaard S, Gårdsvoll H, et al. Peptide-derived antagonists of the urokinase receptor. Affinity maturation by combinatorial chemistry, identification of functional epitopes, and inhibitory effect on cancer cell intravasation. Biochemistry. 2001;40(40):12157-12168.

14. Baldini E, Sorrenti S, D’Armiento E, Di Matteo FM, Catania A, Ulisse S. The urokinase plasminogen activating system in thyroid cancer: clinical implications. G Chir. 2012;33(10):305-310.

15. Mekkawy AH, Morris DL, Pourgholami MH. Urokinase plasminogen activator system as a potential target for cancer therapy. Future Oncol. 2009;5(9):1487-1499.

16. Romer J, Nielsen BS, Ploug M. The urokinase receptor as a potential target in cancer therapy. Curr Pharm Des. 2004;10(19):2359-2376.

17. Yang L, Sajja HK, Cao Z, et al. uPAR-targeted optical imaging contrasts as theranostic agents for tumor margin detection. Theranostics. 2013;4(1): 106-118.

18. Yang L, Peng XH, Wang YA, et al. Receptor-targeted nanoparticles for in vivo imaging of breast cancer. Clin Cancer Res. 2009;15(14): $4722-4732$

19. Liu D, Overbey D, Watkinson L, Giblin MF. Synthesis and characterization of an (111)In-labeled peptide for the in vivo localization of human cancers expressing the urokinase-type plasminogen activator receptor (uPAR). Bioconjug Chem. 2009;20(5):888-894.

20. Wang F, Eric Knabe W, Li L, et al. Design, synthesis, biochemical studies, cellular characterization, and structure-based computational studies of small molecules targeting the urokinase receptor. Bioorg Med Chem. 2012;20(15):4760-4773.

21. Li R, Zheng K, Hu P, et al. A novel tumor targeting drug carrier for optical imaging and therapy. Theranostics. 2014;4(6):642-659.

22. Lee GY, Qian WP, Wang L, et al. Theranostic nanoparticles with controlled release of gemcitabine for targeted therapy and MRI of pancreatic cancer. ACS Nano. 2013;7(3):2078-2089.

23. Patterson TA, Twaddle NC, Roegge CS, Callicott RJ, Fisher JW, Doerge DR. Concurrent determination of bisphenol A pharmacokinetics in maternal and fetal rhesus monkeys. Toxicol Appl Pharmacol. 2013;267(1):41-48.

24. Vom Saal FS, VandeVoort CA, Taylor JA, Welshons WV, Toutain PL, Hunt PA. Bisphenol A (BPA) pharmacokinetics with daily oral bolus or continuous exposure via silastic capsules in pregnant rhesus monkeys: relevance for human exposures. Reprod Toxicol. 2014;45:105-116.

25. Fisher JW, Twaddle NC, Vanlandingham M, Doerge DR. Pharmacokinetic modeling: prediction and evaluation of route dependent dosimetry of bisphenol A in monkeys with extrapolation to humans. Toxicol Appl Pharmacol. 2011;257(1):122-136.

26. Yang $\mathrm{P}$, Han $\mathrm{P}$, Hou J, et al. Electrocardiographic characterization of rhesus monkey model of ischemic myocardial infarction induced by left anterior descending artery ligation. Cardiovasc Toxicol. 2011;11(4): 365-372.

27. Taylor JA, Vom Saal FS, Welshons WV, et al. Similarity of bisphenol A pharmacokinetics in rhesus monkeys and mice: relevance for human exposure. Environ Health Perspect. 2011;119(4):422-430.

28. Chen H, Wang L, Yu Q, et al. Anti-HER2 antibody and ScFvEGFRconjugated antifouling magnetic iron oxide nanoparticles for targeting and magnetic resonance imaging of breast cancer. Int J Nanomedicine. 2013;8:3781-3794.
29. Bulte JW, Kraitchman DL. Iron oxide MR contrast agents for molecular and cellular imaging. NMR Biomed. 2004;17(7):484-499.

30. Thorek DL, Chen AK, Czupryna J, Tsourkas A. Superparamagnetic iron oxide nanoparticle probes for molecular imaging. Ann Biomed Eng. 2006;34(1):23-38.

31. Veiseh O, Gunn JW, Zhang M. Design and fabrication of magnetic nanoparticles for targeted drug delivery and imaging. Adv Drug Deliv Rev. 2010;62(3):284-304.

32. Jun YW, Lee JH, Cheon J. Chemical design of nanoparticle probes for high-performance magnetic resonance imaging. Angew Chem Int Ed Engl. 2008;47(28):5122-5135.

33. Huang J, Zhong X, Wang L, Yang L, Mao H. Improving the magnetic resonance imaging contrast and detection methods with engineered magnetic nanoparticles. Theranostics. 2012;2(1):86-102.

34. Xie J, Chen K, Huang J, et al. PET/NIRF/MRI triple functional iron oxide nanoparticles. Biomaterials. 2010;31(11):3016-3022.

35. Alarifi S, Ali D, Al-Doaiss AA, Ali BA, Ahmed M, Al-Khedhairy AA. Histologic and apoptotic changes induced by titanium dioxide nanoparticles in the livers of rats. Int J Nanomedicine. 2013;8:3937-3943.

36. Cawello W, Fichtner A, Boekens H, Braun M. Influence of hepatic impairment on the pharmacokinetics of the dopamine agonist rotigotine. Eur J Drug Metab Pharmacokinet. 2014;39(3):155-163.

37. Concepcion Navarro M, Pilar Montilla M, Martin A, Jimenez J, Pilar Utrilla M. Free radical scavenger and antihepatotoxic activity of Rosmarinus tomentosus. Planta Med. 1993;59(4):312-314.

38. Kim TY, Kim DJ. Acute-on-chronic liver failure. Clin Mol Hepatol. 2013; 19(4):349-359.

39. Zamora Nava LE, Aguirre Valadez J, Chavez-Tapia NC, Torre A. Acuteon-chronic liver failure: a review. Ther Clin Risk Manag. 2014;10: 295-303.

40. Khare P, Sonane M, Pandey R, Ali S, Gupta KC, Satish A. Adverse effects of TiO2 and $\mathrm{ZnO}$ nanoparticles in soil nematode, Caenorhabditis elegans. J Biomed Nanotechnol. 2011;7(1):116-117.

41. Maiti S. Nanotoxicity of gold and iron nanoparticles. J Biomed Nanotechnol. 2011;7(1):65.

42. You J, Zhou J, Zhou M, et al. Pharmacokinetics, clearance, and biosafety of polyethylene glycol-coated hollow gold nanospheres. Part Fibre Toxicol. 2014;11:26.

43. Sarhan OM, Hussein RM. Effects of intraperitoneally injected silver nanoparticles on histological structures and blood parameters in the albino rat. Int J Nanomedicine. 2014;9:1505-1517.

44. Zheng JC, Lei N, He QC, et al. PEGylation is effective in reducing immunogenicity, immunotoxicity, and hepatotoxicity of alpha-momorcharin in vivo. Immunopharmacol Immunotoxicol. 2012;34(5):866-873.

45. Esmaeillou M, Moharamnejad M, Hsankhani R, Tehrani AA, Maadi H. Toxicity of $\mathrm{ZnO}$ nanoparticles in healthy adult mice. Environ Toxicol Pharmacol. 2013;35(1):67-71.

46. Yang L, Mao H, Cao Z, et al. Molecular imaging of pancreatic cancer in an animal model using targeted multifunctional nanoparticles. Gastroenterology. 2009;136(5):1514-1525.e2.
International Journal of Nanomedicine

\section{Publish your work in this journal}

The International Journal of Nanomedicine is an international, peerreviewed journal focusing on the application of nanotechnology in diagnostics, therapeutics, and drug delivery systems throughout the biomedical field. This journal is indexed on PubMed Central, MedLine, CAS, SciSearch ${ }^{\circledR}$, Current Contents ${ }^{\circledR} /$ Clinical Medicine,
Dovepress

Journal Citation Reports/Science Edition, EMBase, Scopus and the Elsevier Bibliographic databases. The manuscript management system is completely online and includes a very quick and fair peer-review system, which is all easy to use. Visit http://www.dovepress.com/ testimonials.php to read real quotes from published authors. 